\title{
Impact of side branch stenting on five-year long-term clinical outcome with the bifurcation-dedicated Axxess Biolimus A9- eluting stent system
}

\author{
Stefan Verheye ${ }^{1 *}, \mathrm{MD}, \mathrm{PhD}$; Ian Buysschaert ${ }^{2}, \mathrm{MD}, \mathrm{PhD}$; Eberhard Grube ${ }^{3}, \mathrm{MD}, \mathrm{PhD}$ \\ 1. Antwerp Cardiovascular Center, ZNA Middelheim, Antwerp, Belgium;2. ASZ Aalst, Aalst, Belgium; 3. Heart Center, Siegburg, \\ Germany
}

This paper also includes supplementary data published online at: http://www.pcronline.com/eurointervention/91st_issue/176

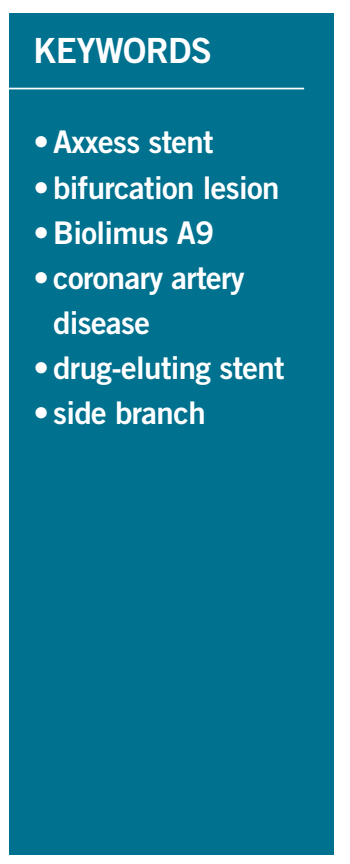

\begin{abstract}
Aims: Our aim was to evaluate the five-year clinical impact of side branch (SB) stenting with a drug-eluting stent (DES) following Axxess stent implantation in coronary bifurcation lesions.

Methods and results: Four hundred patients treated with Axxess were pooled from the AXXESS Plus and DIVERGE five-year follow-up studies. We compared unadjusted and propensity-adjusted major adverse clinical events (MACE) between Axxess with no SB stenting ("Axxess provisional”) versus Axxess with SB stenting ("Axxess additional"). "Axxess additional" had no impact on the MACE rate, with unadjusted and adjusted HR 1.59 (95\% CI: 0.95-2.64) and 1.37 (95\% CI: 0.88-2.13), respectively. No differences were seen in the individual components of death, myocardial infarction and ischaemia-driven target lesion revascularisation, respectively, both in unadjusted (HR 0.92 [95\% CI: 0.38-2.19]; HR 1.73 [95\% CI: 0.783.82]; HR 1.65 [95\% CI: 0.84-3.26]) and adjusted analysis (HR 0.92 [95\% CI: 0.41-2.09]; HR 1.13 [95\% CI: 0.59-2.17]; HR 1.31 [95\% CI: 0.74-2.31]). No differences in definite stent thrombosis were seen with unadjusted HR 2.1 (95\% CI: 0.45-9.88) and adjusted HR 1.0 (95\% CI: 0.32-3.1).
\end{abstract}

Conclusions: Stenting the SB following Axxess implantation does not impact on long-term clinical outcomes compared to MV stenting only. The Axxess stent system offers a safe and tailored alternative for the treatment of coronary bifurcation lesions.

\footnotetext{
*Corresponding author: Antwerp Cardiovascular Institute Middelheim, Ziekenhuis Netwerk Antwerpen, Lindendreef 1, B-2020 Antwerp, Belgium. E-mail: stefan.verheye@gmail.com
} 


\section{Introduction}

Current consensus recommends a single stenting strategy of the main vessel (MV) with bail-out side branch (SB) stenting as the percutaneous treatment strategy of choice for bifurcation lesions ${ }^{1}$. However, this strategy does not apply to all bifurcation lesions, especially when the SB is severely diseased over a long segment. In that case, a double stent technique or dedicated bifurcation device might be the preferred approach. A double stent technique, while being readily available, is technically demanding, time-consuming and sometimes cumbersome. Even if the final result is angiographically perfect, a double stent technique has the micro-anatomical disadvantages of significant strut overlap, crush, distortion or malapposition, all of which might ultimately result in an increased risk of in-stent restenosis, and/or myocardial infarction (MI) driven by stent thrombosis $(\mathrm{ST})^{2}$. Another downside of the double stent technique is the commitment of having to stent both the main and side branches, even if the distal main branch is unaffected.

Dedicated bifurcation devices might solve these issues. However, most devices are complex in nature with asymmetrical designs, requiring a rigid implantation protocol that is often technically demanding, with, for instance, multiple wires, balloons or stents running through the same delivery catheter. The Axxess ${ }^{\mathrm{TM}}$ stent (Biosensors Europe SA, Morges, Switzerland), on the other hand, is a symmetrical self-expanding conically shaped Biolimus A9-eluting stent with a rapid exchange catheter running over a single wire. The device is positioned at the level of the bifurcation carina and flares the ostia of both distal vessels, with no prioritisation of either MV or SB and with no need of stent recrossing, allowing the treatment of both distal branches symmetrically as needed.

Although a double stent strategy using balloon-expandable stents has resulted in mixed results with higher rates of short-term major adverse cardiovascular event (MACE) rates in some ${ }^{3}$, but not all studies ${ }^{4,5}$, one recent large meta-analysis of all current major studies of percutaneous treatment of bifurcation lesions showed an increased risk of long-term late ST and MI after a routine double stent strategy compared to a single stent strategy ${ }^{2}$. Given the advantages of the Axxess stent over conventional balloon-expandable stents in terms of symmetry and equivocal access to both MV and SB resulting in no strut deformation or crush, we compared the five-year long-term impact of additional SB stenting following Axxess implantation in the main vessel in the AXXESS Plus ${ }^{6}$ and DIVERGE ${ }^{7,8}$ studies.

\section{Editorial, see page 850}

\section{Methods}

\section{STUDY POPULATION}

The data from two independent studies were pooled at patient level for the present analysis. Both the AXXESS Plus and DIVERGE studies are prospective, single-arm, multicentre studies of the treatment of bifurcation lesions with the Axxess stent at clinical sites in Europe, Australia, New Zealand and Brazil. The 139 patients in AXXESS Plus were included from July 2004 to February 2005, and the 302 patients in DIVERGE were enrolled from June 2006 to October 2007. Local ethics committees approved the protocols and all patients gave written informed consent before the procedure. The methods with inclusion and exclusion criteria have been published previously ${ }^{6,7}$. In brief, patients of 18 to 80 years of age with documented stable angina, unstable angina or positive functional study and a de novo native bifurcation lesion in a major coronary artery in which either the MV or SB had at least a $50 \%$ stenosis based on visual estimation were included. The reference vessel diameter had to be 2.75 to $3.75 \mathrm{~mm}$ in the MV and $\geq 2.2 \mathrm{~mm}$ in the SB by visual estimate, with a bifurcation angle $<70^{\circ}$ in DIVERGE.

\section{AXXESS STENT AND PROCEDURE}

The Axxess stent system has been described in detail previously $^{9,10}$. It is a self-expanding, conically shaped nitinol stent with a strut thickness of 160 microns, eluting Biolimus A9, a highly lipophilic, semi-synthetic sirolimus analogue (Biosensors Europe SA). The drug is emulsed into a biodegradable PLA polymer applied primarily to the abluminal stent surface. The Biolimus A9 elution half-life is 21 days, and the PLA biodegradable coating is absorbed after six to nine months. A straight configuration of the Axxess stent was available only in AXXESS Plus ${ }^{6}$. The stent diameters available were $2.5,3.0$ and $3.5 \mathrm{~mm}$, with lengths of 11,14 or $20 \mathrm{~mm}$, depending on the study. Not all diameters and lengths were available in both studies. Adjunctive stenting was permitted in the distal MV and SB, at the discretion of the operator. In AXXESS Plus ${ }^{6}$, the protocol allowed the use of commercially available stents in conjunction with the Axxess stent, while the protocol in DIVERGE ${ }^{7}$ mandated the use of the CYPHER ${ }^{\circledR}$ sirolimus-eluting stent (Cordis, Johnson \& Johnson, Warren, NJ, USA). Aspirin ( $\geq 80 \mathrm{mg} /$ day) was given daily and clopidogrel (loading dose $\geq 300 \mathrm{mg}$ and $75 \mathrm{mg} /$ day thereafter) was administered for at least six months in all patients.

\section{POOLED STUDY POPULATION}

Given the similar clinical and angiographic inclusion and exclusion criteria and long-term clinical outcome of both the AXXESS Plus and DIVERGE studies, the two data sets were pooled for analysis. For the sake of uniformity, we excluded the AXXESS Plus patients with the following characteristics: (i) additional stent different from CYPHER (11 patients), (ii) left main lesion (six patients), (iii) patients treated with bare metal stents (three patients), and (iv) those treated with a straight-shaped Axxess stent (18 patients). A total of 35 patients with one or more of these characteristics were excluded. In addition, two patients in AXXESS Plus and four in DIVERGE did not receive any Axxess stent, and were therefore also excluded.

\section{STUDY ENDPOINTS}

Patients were evaluated clinically in both studies with annual contacts up to five years after the procedure. All data were submitted to a coordinating centre (Cardiovascular Research Foundation, New York, NY, USA). A data safety and monitoring board (DSMB) 
periodically reviewed safety data, and all clinical endpoints were adjudicated by an independent clinical events committee (CEC).

The primary endpoint of the present report was the rate of MACE at five years, consisting of any death, myocardial infarction (Q-wave or non-Q-wave), and ischaemia-driven TLR, as previously defined $^{6,7}$. Differences between the two trials in terms of endpoint definition were adjusted and harmonised for the pooled analysis. Secondary safety endpoints were definite or probable ST at five years, according to the Academic Research Consortium criteria ${ }^{11}$.

\section{Statistical analysis}

Continuous variables are presented as mean \pm standard deviation and categorical variables are presented as counts and frequencies. All reports of the individual AXXESS Plus and DIVERGE studies are according to the intention-to-treat principle.

Unadjusted analyses were performed using a Cox proportional hazards model. Adjustment for differences in clinical variables was performed using propensity score matching. A propensity score for each patient was obtained from a logistic regression by inverse probability of treatment weights, predicted from variables associated with severity of coronary artery disease and including: age, sex, weight, body mass index, diabetes status, hypertension, hypercholesterolaemia, angina status, smoking status, renal insufficiency at screening, heart failure, peripheral vascular disease, history of coronary artery bypass graft (CABG), history of percutaneous coronary intervention (PCI) and history of MI. All analyses were performed using SAS version 9.3 (SAS Institute, Cary, NC, USA).

\section{Results \\ DEMOGRAPHICS AND LESION CHARACTERISTICS}

The patient characteristics and locations of stenoses of the complete AXXESS Plus and DIVERGE studies have been published previously ${ }^{6,7}$ and are summarised in Online Table 1 and Online Table 2.

A total of 102 patients from AXXESS Plus and 298 patients from DIVERGE were pooled for a sample size of 400 patients. Baseline characteristics for the individual studies and for the total group are shown in Table 1. Most of the patients were male $(74.9 \%)$, with a mean age of 63.1 years, and presence of diabetes

Table 1. Baseline characteristics of the pooled analysis.

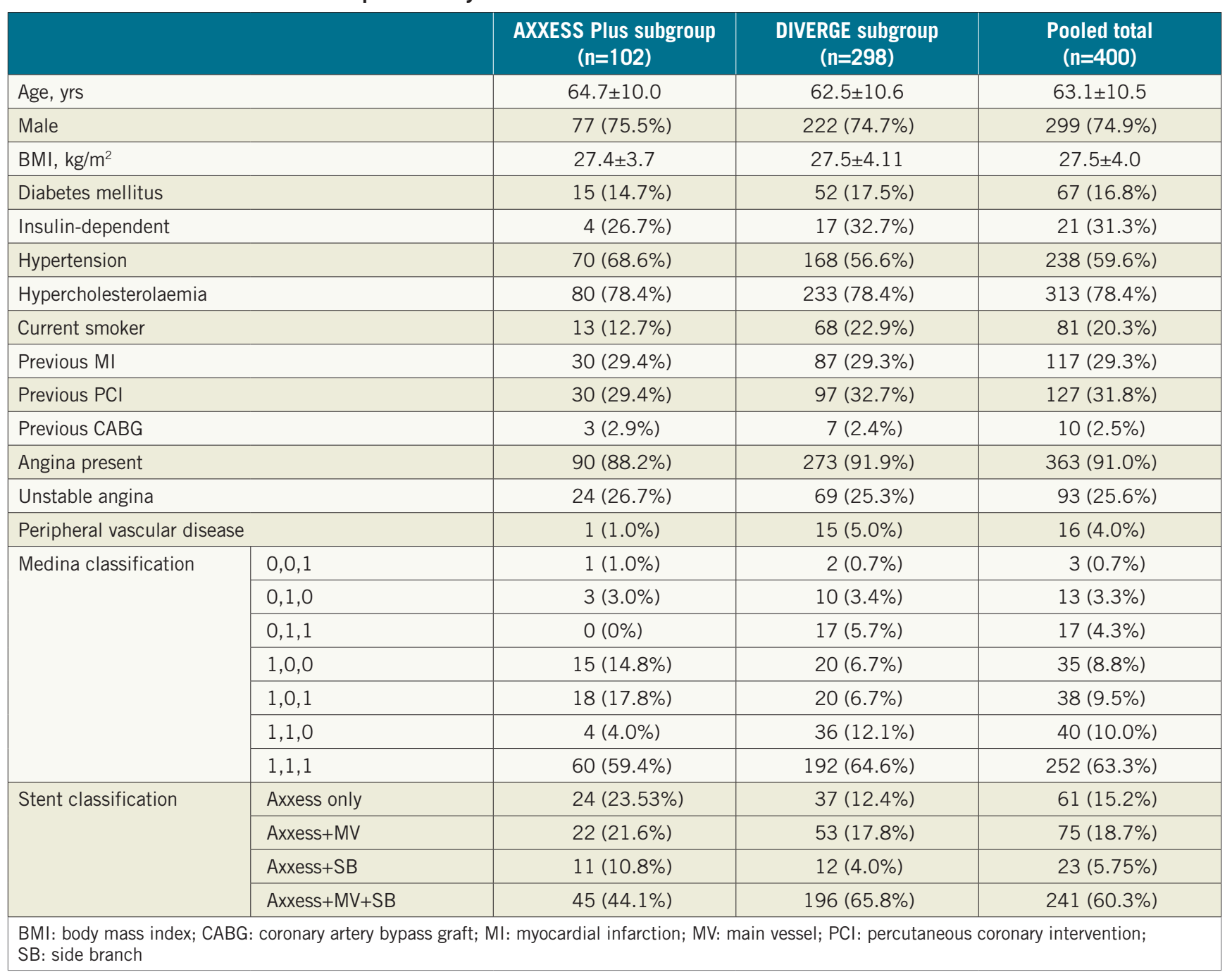


mellitus in $16.8 \%$. The majority of the lesions $(77.1 \%)$ were classified as true bifurcations according to the Medina classification ${ }^{12}$.

Stenting of the MV only, with either an Axxess stent alone or with an Axxess stent and an additional stent in the distal MV but not in the SB, resembles the provisional approach and was termed "Axxess provisional". Stenting of the MV with an Axxess stent and an additional stent in the SB, regardless of whether the distal MV was stented or not, resembles the double stent approach and was termed "Axxess additional". An "Axxess additional" stent implantation was performed in $66.0 \%$ of the patients. Lesion characteristics analysed by quantitative coronary analysis (QCA) are shown in Table 2.

\section{LONG-TERM CLINICAL OUTCOME OF IMPACT OF SB STENTING OR "AXXESS ADDITIONAL"}

The cumulative long-term clinical outcomes of the complete AXXESS Plus and DIVERGE studies are depicted in Online Table 3 and Online Table 4, respectively. In the pooled analysis, the five-year long-term follow-up was slightly higher for DIVERGE (96.3\%) than for AXXESS Plus (86.3\%), with an overall five-year follow-up available for $93.7 \%$ of the pooled patients. As reported in Table 3, outcome after "Axxess additional" was similar to "Axxess provisional". Although the rates of MACE and

A Adjusted MACE events for "Axxess provisional"vs. "Axxess additional"

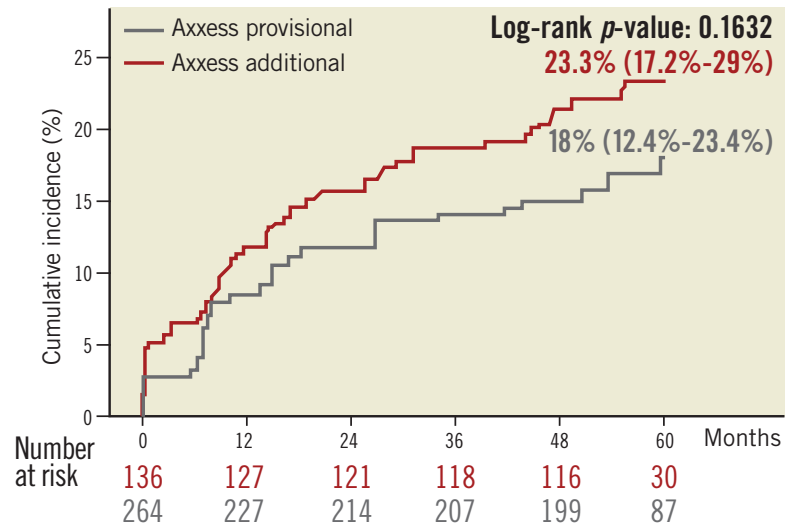

C Adjusted MI events for "Axxess provisional"vs. "Axxess additional"

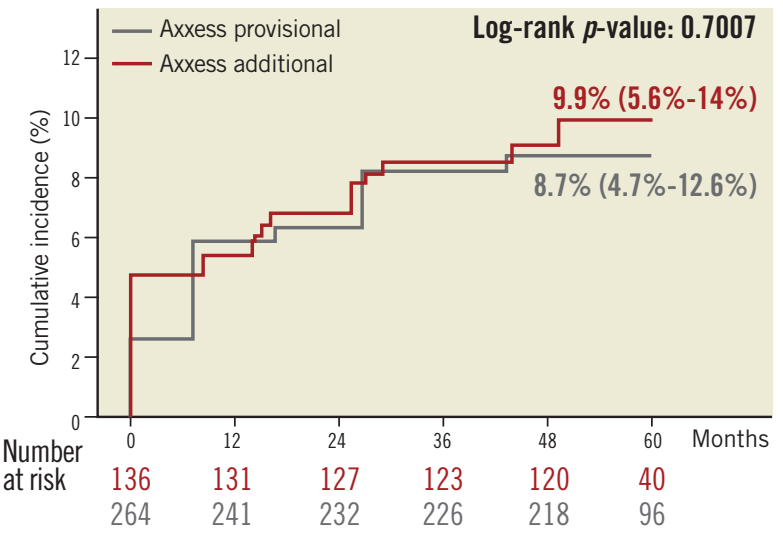

some of its individual components were numerically lower in the "Axxess provisional" group compared to the "Axxess additional" group, none of the differences was statistically significant, both in the unadjusted and propensity analysis, as reported in Table $\mathbf{3}$. Adjusted Kaplan-Meier curves for both "Axxess provisional" and "Axxess additional" groups are shown in Figure 1. In terms of stent thrombosis, no significant differences were seen, as shown in Table 3. A representative case of "Axxess provisional" with medium-term angiographic follow-up is shown in Figure 2.

\section{Discussion}

The present analysis reports the absence of clinical impact of SB stenting following Axxess stent implantation (or "Axxess additional") on the five-year long-term clinical outcome when compared to a provisional-like strategy (or "Axxess provisional") in the percutaneous treatment of de novo coronary bifurcation lesions. Both unadjusted and propensity-adjusted comparison between the two groups in a total of 400 patients pooled from two prospective studies, AXXESS Plus ${ }^{6}$ and DIVERGE ${ }^{7,8}$, found no significant differences in terms of MACE and its individual components of death, MI and ischaemia-driven TLR.

PCI of bifurcation lesions with balloon-expandable stents often challenges the operator with the dilemma as to whether to use

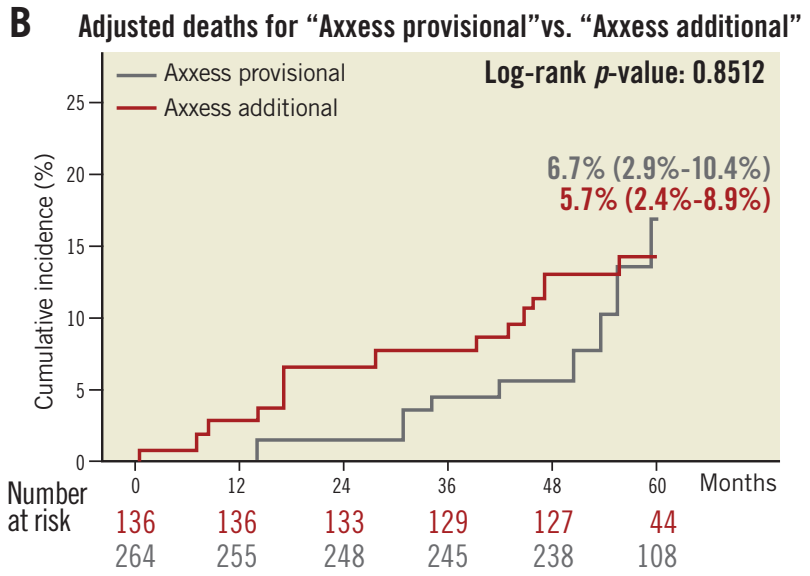

D Adjusted TLR events for "Axxess provisional"vs. "Axxess additional"

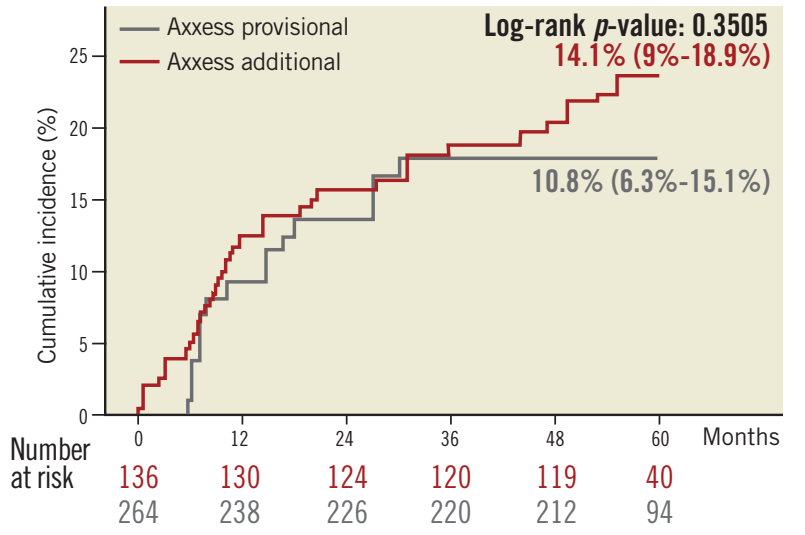

Figure 1. Adjusted Kaplan-Meier curves for "Axxess provisional” versus "Axxess additional” over five years. A) Total MACE. B) Death. C) MI. D) Ischaemia-driven (id)-TLR. 
Table 2. Quantitative coronary analysis.

\begin{tabular}{|l|c|c|c|c|c|c|}
\hline \multirow{2}{*}{} & \multicolumn{2}{|c|}{ Pooled } & \multicolumn{2}{c|}{ "Axxess provisional" } & \multicolumn{2}{c|}{ "Axxess additional" } \\
\cline { 2 - 7 } & $\begin{array}{c}\text { Parent vessel } \\
\mathbf{n = 3 9 8}\end{array}$ & $\begin{array}{c}\text { Side branch } \\
\mathbf{n = 3 9 8}\end{array}$ & $\begin{array}{c}\text { Parent vessel } \\
\mathbf{n = 1 3 6}\end{array}$ & $\begin{array}{c}\text { Side branch } \\
\mathbf{n = 1 3 6}\end{array}$ & $\begin{array}{c}\text { Parent vessel } \\
\mathbf{n = 2 6 2}\end{array}$ & $\begin{array}{c}\text { Side branch } \\
\mathbf{n = 2 6 2}\end{array}$ \\
\hline Reference vessel diameter $(\mathrm{mm})$ & $2.91 \pm 0.36$ & $2.31 \pm 0.33$ & $2.88 \pm 0.36$ & $2.25 \pm 0.33$ & $2.91 \pm 0.36$ & $2.34 \pm 0.33$ \\
\hline Minimal luminal diameter $(\mathrm{mm})$ & $0.84 \pm 0.36$ & $0.99 \pm 0.54$ & $0.84 \pm 0.36$ & $1.32 \pm 0.66$ & $0.84 \pm 0.36$ & $0.81 \pm 0.39$ \\
\hline Diameter stenosis (\%) & $71.2 \pm 11.2$ & $57.5 \pm 21.7$ & $71.1 \pm 11.7$ & $42.4 \pm 25.2$ & $71.2 \pm 11.1$ & $65.2 \pm 14.7$ \\
\hline Lesion length (mm) & $15.1 \pm 6.8$ & $7.1 \pm 3.5$ & $13.9 \pm 7.2$ & $5.5 \pm 1.9$ & $15.8 \pm 6.5$ & $7.7 \pm 3.7$ \\
\hline
\end{tabular}

a single stent or a double stent strategy. While the provisional technique is the preferred strategy whenever possible, bail-out stenting of the SB is sometimes needed, certainly in case of reduced or occluded flow in a large SB following MV stenting. This step is often time-consuming and technically demanding. Recrossing the stent towards the SB, preferably through the most distal cell, can be challenging and sometimes even impossible, while requiring extra resources in terms of wires and balloons to pass and dilate the struts. Another potential problem of this bail-out double stent approach is that not all double stent strategies are applicable once the MV has been stented first. The crush and mini-crush techniques, for instance, can no longer be applied and have to be replaced by an internal crush which leaves crushed struts intraluminally instead of abluminally ${ }^{13}$.

In contrast to this is the simple and tailored approach with the Axxess strategy. The advantage is that the same technique is used to tackle any bifurcation lesion, with no upfront commitment to additional SB or main branch stenting, and with no prioritisation
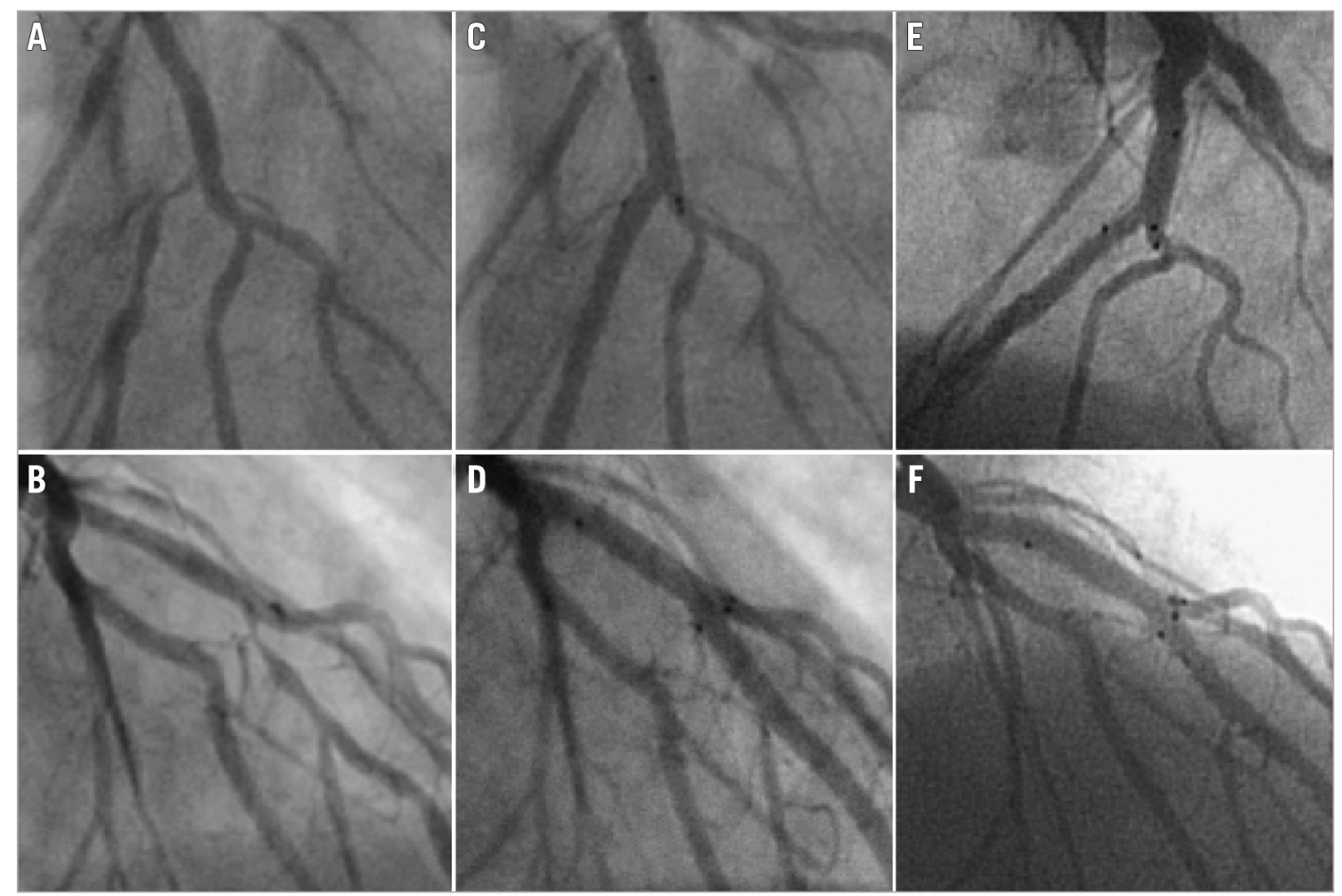

Figure 2. Case example after "Axxess provisional" stent implantation. Sequential coronary angiogram magnifications of a 53-year-old active smoker with dyslipidaemia and presenting with angina due to a severe stenosis of the left anterior descending artery at the bifurcation with the first diagonal branch. The patient was successfully treated with the Axxess stent and an additional CYPHER stent distal in the MV. At 19 months post procedure, he presented with unstable angina. Coronary angiography showed no in-stent restenosis or ST. Diffuse coronary spasms were noted during angiography, which responded to intracoronary nitrates. He was diagnosed with variant angina and treated with calcium antagonists and nitrates. A) \& B) Baseline angiograms after intracoronary nitrates showing a severe stenosis of the left anterior descending artery, starting at the bifurcation with the first diagonal branch (Medina 0,1,0). C) \& D) Final angiographic results after PCI with Axxess stent $(3.0 \times 14 \mathrm{~mm})$, and additional CYPHER stents distal in the LAD $(2.75 \times 28 \mathrm{~mm})$. E) \& F) Angiographic results at $19 \mathrm{months}$ post procedure showing patent stents with no sign of restenosis or thrombosis. Panels A, C and E are in LAO-cranial projections and lower panels $B, D$ and $F$ are in $R A O$-cranial projections. 
Table 3. Cumulative clinical outcomes per stent group at five-year follow-up.

\begin{tabular}{|c|c|c|c|c|c|c|c|}
\hline \multirow{2}{*}{\multicolumn{2}{|c|}{ n (\%) }} & \multirow{2}{*}{$\begin{array}{c}\text { "Axxess } \\
\text { provisional" } \\
n=136(34.0 \%)\end{array}$} & \multirow{2}{*}{$\begin{array}{c}\text { "Axxess } \\
\text { additional" } \\
\text { n=264 (66.0\%) }\end{array}$} & \multicolumn{2}{|c|}{ Unadjusted } & \multicolumn{2}{|c|}{ Propensity analysis } \\
\hline & & & & Hazard ratio & $p$-value & Hazard ratio & $p$-value \\
\hline \multicolumn{2}{|c|}{ MACE (death, MI, id-TLR) } & $20(14.7 \%)$ & $59(22.3 \%)$ & $1.59(0.95-2.64)$ & 0.07 & $1.37(0.88-2.13)$ & 0.16 \\
\hline \multicolumn{2}{|l|}{ Death } & $8(5.9 \%)$ & $14(5.3 \%)$ & $0.92(0.38-2.19)$ & 0.85 & $0.92(0.41-2.09)$ & 0.85 \\
\hline \multicolumn{2}{|c|}{ Cardiac death } & $5(3.7 \%)$ & $8(3.0 \%)$ & $0.84(0.28-2.58)$ & 0.77 & $0.97(0.33-2.85)$ & 0.95 \\
\hline \multicolumn{2}{|c|}{ Non-cardiac death } & $3(2.2 \%)$ & $6(2.3 \%)$ & $1.04(0.26-4.16)$ & 0.95 & $0.87(0.25-3.03)$ & 0.82 \\
\hline \multicolumn{2}{|l|}{ MI } & $8(5.9 \%)$ & $26(9.8 \%)$ & $1.73(0.78-3.82)$ & 0.18 & $1.13(0.59-2.17)$ & 0.71 \\
\hline \multicolumn{2}{|l|}{ Q-wave } & $4(2.9 \%)$ & $8(3.0 \%)$ & $1.04(0.31-3.46)$ & 0.94 & $0.77(0.27-2.18)$ & 0.62 \\
\hline \multicolumn{2}{|c|}{ Non-Q-wave } & $4(2.9 \%)$ & $20(7.6 \%)$ & $2.65(0.91-7.77)$ & 0.07 & $1.61(0.70-3.70)$ & 0.26 \\
\hline \multicolumn{2}{|c|}{ Procedure-related MI } & $3(2.2 \%)$ & $12(4.5 \%)$ & $2.08(0.59-7.36)$ & 0.26 & $1.51(0.50-4.55)$ & 0.47 \\
\hline \multicolumn{2}{|c|}{ Ischaemia-driven TLR } & $11(8.1 \%)$ & $35(13.3 \%)$ & $1.65(0.84-3.26)$ & 0.15 & $1.31(0.74-2.32)$ & 0.35 \\
\hline \multicolumn{2}{|l|}{$\mathrm{PCl}$} & $11(8.1 \%)$ & $31(11.7 \%)$ & $1.45(0.73-2.90)$ & 0.29 & $1.17(0.65-2.09)$ & 0.60 \\
\hline \multicolumn{2}{|l|}{ CABG } & $0(0 \%)$ & $6(2.3 \%)$ & $\mathrm{N} / \mathrm{A}$ & $\mathrm{N} / \mathrm{A}$ & $N / A$ & $\mathrm{~N} / \mathrm{A}$ \\
\hline \multirow{3}{*}{$\begin{array}{l}\text { Stent } \\
\text { thrombosis } \\
\text { (ARC def.) }\end{array}$} & Definite & $2(1.5 \%)$ & $8(3.0 \%)$ & $2.10(0.45-9.88)$ & 0.35 & $1.00(0.32-3.1)$ & 0.99 \\
\hline & Probable & $0(0 \%)$ & $2(0.8 \%)$ & $N / A$ & $N / A$ & $N / A$ & N/A \\
\hline & Possible & $4(2.9 \%)$ & $4(1.5 \%)$ & $0.53(0.13-2.12)$ & 0.37 & $0.62(0.17-2.33)$ & 0.48 \\
\hline
\end{tabular}

ARC def.: Academic Research Consortium definition; CABG: coronary artery bypass graft; $\mathrm{Cl}$ : confidence interval; id-TLR: ischaemia-driven target lesion revascularisation; MACE: major adverse cardiac events; MI: myocardial infarction; PCI: percutaneous coronary intervention; TLR: target lesion revascularisation

of any distal branch. The Axxess stent is always deployed first in the parent vessel and will span the carina and flare the ostia of both distal branches. There is no carina shift as in a conventional provisional stenting. Following the Axxess deployment, additional stents can be implanted in any or both distal branches when deemed necessary. There is no need to recross or dilate any struts, and there is no commitment to additional steps once the results are acceptable. This stepwise approach is predictable, with no need for often challenging strut recrossing and ballooning, and results in good short- ${ }^{7}$, medium- ${ }^{8}$ and now long-term clinical outcome, as well as on intravascular ultrasound (IVUS) analysis ${ }^{14}$, as published previously.

While our current long-term clinical outcome results are in line with previously reported randomised comparisons between single and double stent strategies, which reported no clear clinical benefit or detriment from additional SB stenting over provisional stenting ${ }^{4,5}$, it should be noted that few bifurcation studies have published five-year outcome data so far. The present manuscript is the first to report five-year follow-up data of a dedicated bifurcation device, whereas for the balloon-expandable DES only the NORDIC Bifurcation Study has reported five-year follow-up ${ }^{15}$. The long-term results in NORDIC, with a MACE rate of $18.3 \%$ in the optional SB stenting group and $28.2 \%$ in the MV and SB stenting group $(\mathrm{p}=0.03)$, were higher than those reported here, with adjusted Kaplan-Meier estimates of $18.0 \%$ and $23.3 \%$, respectively. It is worth noting, however, that procedure-related MI were excluded in the NORDIC combined endpoint, while they were not in AXXESS Plus and DIVERGE and in the pooled analysis. When excluding periprocedural MI in our data, we found even lower numbers with a MACE rate of $17.0 \%$ in the "Axxess provisional" group and $19.9 \%$ in the "Axxess additional" group. Hence, the present report confirms that the easiness of the Axxess stent and its tailored strategy with regard to the additional stenting of the distal vessels also results in excellent long-term clinical outcome with no impact of additional stenting.

The same holds true for stent thrombosis. A recent metaanalysis of randomised and observational studies with a total of 6,961 patients reported that an SB stent strategy was associated with an increased risk of definite stent thrombosis and MI, with, respectively, RR of 2.31 (95\% CI: 1.33-4.03) and 1.86 (95\% CI: 1.34-2.60 $)^{2}$. However, we found no differences in definite ST after SB stenting, with adjusted HR 1.0 (95\% CI: 0.32-3.1) for "Axxess additional" compared to "Axxess provisional". The Axxess system therefore seems to be safe, again with no impact of SB stenting.

The size of the SB has recently become of interest as a selection parameter for a two-stent approach. It could be argued that we found no differences between the "Axxess provisional" and "Axxess additional" groups because of too small SB reference diameters. While the majority of the earlier bifurcation trials such as CACTUS, BBC and the NORDIC trials found no major differences between a one- and a two-stent strategy in terms of target vessel failure $^{16}$, the more recent DKCRUSH-II trial showed that the complex DK-crush technique had less need for revascularisation inside the side branches compared to provisional stenting ${ }^{17}$. These favourable results were attributed to the enrolment of patients with bifurcation lesions with larger visually estimated SB size of $\geq 2.5 \mathrm{~mm}$. However, mean RVD of the SB in DKCRUSH-II ${ }^{17}$, $2.29 \pm 0.35 \mathrm{~mm}$ in one-stent and $2.38 \pm 0.32 \mathrm{~mm}$ in two-stent, is not larger than other trials (e.g., in $\mathrm{NORDIC}^{4}, 2.24 \pm 0.46$ vs. $2.28 \pm 0.51 \mathrm{~mm})$ or in our present study $(2.31 \pm 0.33 \mathrm{~mm})$. Similarly, 
selection criteria for inclusion in the recent Tryton IDE trial were bifurcation lesions with visually estimated SB size of $\geq 2.5 \mathrm{~mm}$, while the mean SB size of the effectively included lesions was $2.21 \pm 0.33 \mathrm{~mm}$ in the one-stent group vs. $2.25 \pm 0.30 \mathrm{~mm}$ in the twostent group ${ }^{18}$. Our observed signal cannot therefore be explained by the selection of bifurcation lesions with too small SB.

Many dedicated bifurcation stents have been developed so $\operatorname{far}^{18-20}$. However, none has been widely adopted in the cathlab as yet. On a general level, most dedicated devices are limited to particular types of bifurcations, and do not adhere to the "one device fits all" principle. In addition, the majority of the dedicated devices are hampered by a complex and asymmetrical design, implicating a more challenging positioning, resulting in a higher operator skills requirement and a lower success rate. Also, a commitment to stent both branches is often made even if not warranted, with some devices only being available in a bare metal version. None of these is applicable to the Axxess stent. It is by nature a single and symmetrical DES, which is implanted on a single wire positioned either in the MV or SB. It can be used for all bifurcation types, with the only limitation being a bifurcation angle of $70^{\circ}$ or less. While both distal branches can be treated with additional stents if needed, there is no commitment to doing so if not necessary. Hence, we believe the Axxess stent system offers a flexible alternative for bifurcation stenting.

\section{Study limitations}

The strong points of the studies are the prospective, very longterm follow-up, with independent adjudication of the clinical events, and periodic review of the data by an independent data safety monitoring board. The limitations, however, are the nonrandomised and non-"all-comers" design, as well as the exclusion of patients treated with BMS or non-commercially available Axxess stents, which implies a degree of selection bias. For instance, patients requiring additional SB stenting might represent a patient subset with a higher degree of disease burden, and the non-balanced groups might increase the number of events in the larger group. Furthermore, the use of the Axxess system required implantation of two stents in $75 \%$ of the bifurcation lesions. Although both the AXXESS Plus and DIVERGE studies have a comparable study protocol and patient population, a certain amount of heterogeneity is inherent. In addition, while propensity score matching can correct for potential confounders, it cannot eliminate them completely: this analysis is hence inferior to a randomised trial. Finally, given the relatively small sample size, the present study is underpowered and should be considered as hypothesis-generating.

\section{Conclusions}

The treatment of bifurcation lesions with SB stenting following Axxess stent implantation in the MV does not impact on the fiveyear long-term clinical outcome in terms of MACE, or in terms of ST. The Axxess stent system therefore offers a convincing alternative for the tailored treatment of coronary bifurcation lesions.

\section{Impact on daily practice}

Both simple and complex coronary artery bifurcations can be treated with the Axxess stent followed by additional drug-eluting stents in one or both distal branches. The present study showed that five-year long-term clinical outcomes are good, with similar MACE rates compared to randomised bifurcation trials with long-term follow-up. Stenting the side branch following Axxess implantation (resembling a double stent technique) did not impact on the long-term clinical outcomes when compared to main vessel stenting only (resembling provisional stenting). A complete reconstruction of the bifurcation can therefore be achieved, with minimal stent overlap or distortion, and with good long-term clinical outcome.

\section{Acknowledgements}

The contributions of Samuel Copt (statistical analyses) and of Susanne Meis (careful review of the paper), both full-time employees at Biosensors, are gratefully acknowledged.

\section{Funding}

The study was supported by Biosensors International, Morges, Switzerland.

\section{Conflict of interest statement}

The authors have no conflicts of interest to declare.

\section{References}

1. Lassen JF, Holm NR, Stankovic G, Lefevre T, Chieffo A, Hildick-Smith D, Pan M, Darremont O, Albiero R, Ferenc M, Louvard Y. Percutaneous coronary intervention for coronary bifurcation disease: consensus from the first 10 years of the European Bifurcation Club meetings. EuroIntervention. 2014;10:545-60.

2. Zimarino M, Corazzini A, Ricci F, Di Nicola M, De Caterina R. Late thrombosis after double versus single drug-eluting stent in the treatment of coronary bifurcations: a meta-analysis of randomized and observational Studies. JACC Cardiovasc Interv. 2013;6:687-95.

3. Hildick-Smith D, de Belder AJ, Cooter N, Curzen NP, Clayton TC, Oldroyd KG, Bennett L, Holmberg S, Cotton JM, Glennon PE, Thomas MR, Maccarthy PA, Baumbach A, Mulvihill NT, Henderson RA, Redwood SR, Starkey IR, Stables RH. Randomized trial of simple versus complex drug-eluting stenting for bifurcation lesions: the British Bifurcation Coronary Study: old, new, and evolving strategies. Circulation. 2010;121:1235-43.

4. Steigen TK, Maeng M, Wiseth R, Erglis A, Kumsars I, Narbute I, Gunnes P, Mannsverk J, Meyerdierks O, Rotevatn S, Niemela M, Kervinen K, Jensen JS, Galloe A, Nikus K, Vikman S, Ravkilde J, James S, Aaroe J, Ylitalo A, Helqvist S, Sjogren I, Thayssen P, Virtanen K, Puhakka M, Airaksinen J, Lassen JF, Thuesen L; Nordic PCI Study Group. Randomized study on simple versus complex stenting of coronary artery bifurcation lesions: the Nordic bifurcation study. Circulation. 2006;114:1955-61. 
5. Colombo A, Bramucci E, Sacca S, Violini R, Lettieri C, Zanini R, Sheiban I, Paloscia L, Grube E, Schofer J, Bolognese L, Orlandi M, Niccoli G, Latib A, Airoldi F. Randomized study of the crush technique versus provisional side-branch stenting in true coronary bifurcations: the CACTUS (Coronary Bifurcations: Application of the Crushing Technique Using Sirolimus-Eluting Stents) Study. Circulation. 2009;119:71-8.

6. Grube E, Buellesfeld L, Neumann FJ, Verheye S, Abizaid A, McClean D, Mueller R, Lansky A, Mehran R, Costa R, Gerckens U, Trauthen B, Fitzgerald PJ. Six-month clinical and angiographic results of a dedicated drug-eluting stent for the treatment of coronary bifurcation narrowings. Am J Cardiol. 2007;99:1691-7.

7. Verheye S, Agostoni P, Dubois CL, Dens J, Ormiston J, Worthley S, Trauthen B, Hasegawa T, Koo BK, Fitzgerald PJ, Mehran R, Lansky AJ. 9-month clinical, angiographic, and intravascular ultrasound results of a prospective evaluation of the Axxess self-expanding biolimus A9-eluting stent in coronary bifurcation lesions: the DIVERGE (Drug-Eluting Stent Intervention for Treating Side Branches Effectively) study. J Am Coll Cardiol. 2009;53:1031-9.

8. Buysschaert I, Dubois CL, Dens J, Ormiston J, Worthley S, McClean D, Ottervanger JP, Meredith I, Uren N, Wijns W, Whitbourn R, Mehran R, Lansky AJ, Bichalska M, Meis S, Verheye S. Three-year clinical results of the Axxess Biolimus A9 eluting bifurcation stent system: the DIVERGE study. EuroIntervention. 2013;9:573-81.

9. Verheye S, Trauthen B. Axxess Biolimus A9(R) eluting bifurcation stent system. EuroIntervention. 2007;2:506-8.

10. Dubois CL, Wijns W. The AXXESSTM self-expanding biolimus A9тм eluting stent system for coronary bifurcation lesions. EuroIntervention. 2010;6 Suppl J:J130-4.

11. Cutlip DE, Windecker S, Mehran R, Boam A, Cohen DJ, van Es GA, Steg PG, Morel MA, Mauri L, Vranckx P, McFadden E, Lansky A, Hamon M, Krucoff MW, Serruys PW; Academic Research Consortium. Clinical end points in coronary stent trials: a case for standardized definitions. Circulation. 2007;115: 2344-51.

12. Medina A, Suarez de Lezo J, Pan M. [A new classification of coronary bifurcation lesions]. [Article in Spanish]. Rev Esp Cardiol. 2006;59:183.

13. Louvard Y, Medina A, Stankovic G. Definition and classification of bifurcation lesions and treatments. EuroIntervention. 2010;6 Suppl J:J31-5.

14. Buysschaert I, Sanidas E, Hasegawa T, Koo BK, Honda Y, Fitzgerald PJ, Verheye S. Baseline and 9 months IVUS analysis of the bifurcation-dedicated biolimus A9-eluting Axxess stent system: the DIVERGE IVUS substudy. Catheter Cardiovasc Interv. 2014;84:1062-70.

15. Maeng M, Holm NR, Erglis A, Kumsars I, Niemela M, Kervinen K, Jensen JS, Galloe A, Steigen TK, Wiseth R, Narbute I, Gunnes P, Mannsverk J, Meyerdierks O, Rotevatn S, Nikus K, Vikman S, Ravkilde J, James S, Aaroe J, Ylitalo A, Helqvist S,
Sjogren I, Thayssen P, Virtanen K, Puhakka M, Airaksinen J, Christiansen EH, Lassen JF, Thuesen L; Nordic-Baltic Percutaneous Coronary Intervention Study Group. Long-term results after simple versus complex stenting of coronary artery bifurcation lesions: Nordic Bifurcation Study 5-year follow-up results. J Am Coll Cardiol. 2013;62:30-4.

16. Gao XF, Zhang YJ, Tian NL, Wu W, Li MH, Bourantas CV, Jiang XM, Wang ZM, Li B, Mao WX, Zhang JJ, Chen SL. Stenting strategy for coronary artery bifurcation with drug-eluting stents: a meta-analysis of nine randomised trials and systematic review. EuroIntervention. 2014;10:561-9.

17. Chen SL, Santoso T, Zhang JJ, Ye F, Xu YW, Fu Q, Kan J, Paiboon C, Zhou Y, Ding SQ, Kwan TW. A randomized clinical study comparing double kissing crush with provisional stenting for treatment of coronary bifurcation lesions: results from the DKCRUSH-II (Double Kissing Crush versus Provisional Stenting Technique for Treatment of Coronary Bifurcation Lesions) trial. J Am Coll Cardiol. 2011;57:914-20.

18. Genereux P, Kumsars I, Lesiak M, Kini A, Fontos G, Slagboom T, Ungi I, Metzger DC, Wykrzykowska JJ, Stella PR, Bartorelli AL, Fearon WF, Lefevre T, Feldman RL, LaSalle L, Francese DP, Onuma Y, Grundeken MJ, Garcia-Garcia HM, Laak LL, Cutlip DE, Kaplan AV, Serruys PW, Leon MB. A randomized trial of a dedicated bifurcation stent versus provisional stenting in the treatment of coronary bifurcation lesions. J Am Coll Cardiol. 2015;65:533-43.

19. Gil RJ, Vassilev D, Michalek A, Kern A, Formuszewicz R, Dobrzycki S, Wojcik J, Lesiak M, Kardaszewicz P, Lekston A. Dedicated paclitaxel-eluting bifurcation stent BiOSS ${ }^{\circledR}$ (bifurcation optimisation stent system): 12-month results from a prospective registry of consecutive all-comers population. EuroIntervention. 2012;8:316-24.

20. Verheye S, Ramcharitar S, Grube E, Schofer JJ, Witzenbichler B, Kovac J, Hauptmann KE, Agostoni P, Wiemer M, Lefevre T, Spaargaren R, Serruys PW, Garcia-Garcia HM, van Geuns RJ. Six-month clinical and angiographic results of the STENTYS ${ }^{\circledR}$ self-apposing stent in bifurcation lesions. EuroIntervention. 2011;7:580-7.

\section{Supplementary data}

Online Table 1. Baseline characteristics in AXXESS Plus and DIVERGE studies.

Online Table 2. Lesion characteristics and stent implantation.

Online Table 3. AXXESS Plus study: cumulative clinical outcomes to five years.

Online Table 4. DIVERGE study: cumulative clinical outcomes to five years.

The supplementary data are published online at:

http://www.pcronline.com/

eurointervention/91st_issue/176 


\section{Supplementary data}

Online Table 1. Baseline characteristics in AXXESS Plus and DIVERGE studies.

\begin{tabular}{|l|c|c|}
\hline Age, yrs & $\begin{array}{c}\text { AXXSS Plus study } \\
\mathbf{n = 1 3 9}\end{array}$ & $\begin{array}{c}\text { DIVERGE study } \\
\mathbf{n = 3 0 2}\end{array}$ \\
\hline Male & $64.4 \pm 10.2$ & $62.8 \pm 10.6$ \\
\hline BMI, kg/m² & $102(73.4 \%)$ & $224(74.2 \%)$ \\
\hline Diabetes mellitus & $27.6 \pm 3.8$ & $27.5 \pm 4.1$ \\
\hline Insulin-dependent & $8(5.8 \%)$ & $17(5.6 \%)$ \\
\hline Hypertension & $102(73.4 \%)$ & $171(56.6 \%)$ \\
\hline Hypercholesterolaemia & $109(78.8 \%)$ & $236(78.1 \%)$ \\
\hline Current smoker & $18(12.9 \%)$ & $71(23.5 \%)$ \\
\hline Previous MI & $43(30.9 \%)$ & $88(29.1 \%)$ \\
\hline Previous PCI & $42(30.2 \%)$ & $96(31.8 \%)$ \\
\hline Previous CABG & $6(4.3 \%)$ & $7(2.3 \%)$ \\
\hline Stable angina & $96(69.1 \%)$ & $207(68.5 \%)$ \\
\hline Unstable angina & $26(18.7 \%)$ & $71(23.5 \%)$ \\
\hline Ejection fraction & $65.74 \pm 13.14$ & $68.4 \pm 11.1$ \\
\hline $\begin{array}{l}\text { Glycoprotein IIb/IIla } \\
\text { inhibitors }\end{array}$ & $19(13.7 \%)$ & $36(11.9 \%)$ \\
\hline $\begin{array}{l}\text { BMI: body mass index; CABG: coronary artery bypass graft; } \\
\text { MI: myocardial infarction; PCI: percutaneous coronary intervention }\end{array}$ \\
\hline
\end{tabular}

Online Table 2. Lesion characteristics and stent implantation.

\begin{tabular}{|c|c|c|}
\hline & $\begin{array}{c}\text { AXXESS } \\
\text { Plus study } \\
n=139\end{array}$ & $\begin{array}{c}\text { DIVERGE } \\
\text { study } \\
n=302\end{array}$ \\
\hline \multicolumn{3}{|l|}{ Target vessel } \\
\hline Left anterior descending/diagonal (LAD/D) & $102(73.4 \%)$ & $244(80.8 \%)$ \\
\hline Circumflex/obtuse marginal (CX/OM) & $24(17.3 \%)$ & $44(14.6 \%)$ \\
\hline Right coronary artery/posterior descending (RCA/PD) & $5(3.6 \%)$ & $14(4.6 \%)$ \\
\hline Left main stem & $8(5.8 \%)$ & - \\
\hline \multicolumn{3}{|l|}{ Stent implantation } \\
\hline MV only & $26(19.1 \%)$ & $37(12.2 \%)$ \\
\hline MV plus distal MV or SB & $53(39.0 \%)$ & $65(21.5 \%)$ \\
\hline MV plus both distal MV and SB & $57(41.9 \%)$ & $194(64.2 \%)$ \\
\hline Bifurcation angle, $^{\circ}$ & N/A & $54.2 \pm 24.9$ \\
\hline
\end{tabular}

Online Table 3. AXXESS Plus study: cumulative clinical outcomes to five years.

\begin{tabular}{|l|r|r|r|r|r|}
\hline \multirow{2}{*}{} & \multicolumn{5}{|c|}{ Cumulative follow-up } \\
\cline { 2 - 5 } N $(\%)$ & 1 year & 2 years & 3 years & 4 years & 5 years \\
\hline MACE (cardiac death, MI, emergent CABG, id-TLR) & $19(14.1 \%)$ & $19(15.6 \%)$ & $21(17.2 \%)$ & $23(18.9 \%)$ & $23(19.7 \%)$ \\
\hline Death & $139(100 \%)$ & $122(87.7 \%)$ & $122(87.7 \%)$ & $122(87.7 \%)$ & $117(84.2 \%)$ \\
\hline Cardiac death & $1(0.7 \%)$ & $1(0.8 \%)$ & $3(2.5 \%)$ & $3(2.5 \%)$ & $4(3.4 \%)$ \\
\hline Non-cardiac death & $1(0.7 \%)$ & $1(0.8 \%)$ & $2(1.6 \%)$ & $2(1.6 \%)$ & $2(1.7 \%)$ \\
\hline MI & $9(6.7 \%)$ & $9(7.4 \%)$ & $10(8.2 \%)$ & $11(9.0 \%)$ & $11(9.4 \%)$ \\
\hline Q-wave & $2(1.5 \%)$ & $2(1.6 \%)$ & $2(1.6 \%)$ & $4(3.3 \%)$ & $4(3.4 \%)$ \\
\hline Non-Q-wave & $7(5.2 \%)$ & $7(5.7 \%)$ & $8(6.6 \%)$ & $8(6.6 \%)$ & $8(6.8 \%)$ \\
\hline Ischaemia-driven TLR & $13(9.6 \%)$ & $13(10.7 \%)$ & $14(11.5 \%)$ & $15(12.3 \%)$ & $15(12.8 \%)$ \\
\hline PCI & $12(8.9 \%)$ & $12(9.8 \%)$ & $13(10.7 \%)$ & $14(11.5 \%)$ & $14(12.0 \%)$ \\
\hline CABG & $1(0.7 \%)$ & $1(0.8 \%)$ & $1(0.8 \%)$ & $1(0.8 \%)$ & $1(0.9 \%)$ \\
\hline Target vessel failure & $21(15.6 \%)$ & $21(17.2 \%)$ & $23(18.9 \%)$ & $25(20.5 \%)$ & $25(21.4 \%)$ \\
\hline Stent thrombosis (ARC def.) & & & & \\
\hline Definite or probable & $3(2.2 \%)$ & $3(2.5 \%)$ & $3(2.5 \%)$ & $3(2.5 \%)$ & $3(2.6 \%)$ \\
\hline Possible & $1(0.7 \%)$ & $1(0.7 \%)$ & $3(2.5 \%)$ & $3(2.5 \%)$ & $4(3.4 \%)$ \\
\hline ARC
\end{tabular}

ARC def.: Academic Research Consortium definition; CABG: coronary artery bypass graft; Cl: confidence interval; id-TLR: ischaemia-driven target lesion revascularisation; MACE: major adverse cardiac events; MI: myocardial infarction; PCl: percutaneous coronary intervention; TLR: target lesion revascularisation 
Online Table 4. DIVERGE study: cumulative clinical outcomes to five years.

\begin{tabular}{|c|c|c|c|c|c|}
\hline \multirow[b]{3}{*}{$\mathbf{N}(\%)$} & \multicolumn{5}{|c|}{ Cumulative follow-up } \\
\hline & 1 year & 2 years & 3 years & 4 years & 5 years \\
\hline & $300(99.3 \%)$ & $300(99.3 \%)$ & $298(98.7 \%)$ & $297(98.3 \%)$ & $291(96.4 \%)$ \\
\hline MACE (death, MI, id-TLR) & $28(9.3 \%)$ & $42(14.0 \%)$ & $48(16.1 \%)$ & $54(18.2 \%)$ & $62(21.3 \%)$ \\
\hline Death & $2(0.7 \%)$ & $7(2.3 \%)$ & $9(3.0 \%)$ & $15(5.1 \%)$ & $19(6.5 \%)$ \\
\hline Cardiac death & $2(0.7 \%)$ & $4(1.3 \%)$ & $6(2.0 \%)$ & $11(3.7 \%)$ & $11(3.8 \%)$ \\
\hline Non-cardiac death & $0(0.0 \%)$ & $3(1.0 \%)$ & $3(1.0 \%)$ & $5(1.3 \%)$ & $8(2.7 \%)$ \\
\hline $\mathrm{MI}$ & $13(4.3 \%)$ & $18(6.0 \%)$ & $22(7.4 \%)$ & $23(7.7 \%)$ & $25(8.6 \%)$ \\
\hline Q-wave & $3(1.0 \%)$ & $7(2.3 \%)$ & $8(2.7 \%)$ & $8(2.7 \%)$ & $9(3.1 \%)$ \\
\hline Non-Q-wave & $10(3.3 \%)$ & $11(3.7 \%)$ & $15(5.0 \%)$ & $16(5.4 \%)$ & $17(5.8 \%)$ \\
\hline Ischaemia-driven TLR & $18(6.0 \%)$ & $26(8.7 \%)$ & $30(10.1 \%)$ & 31 (10.4\%) & $36(12.4 \%)$ \\
\hline $\mathrm{PCl}$ & $15(5.0 \%)$ & $23(7.7 \%)$ & $27(9.1 \%)$ & $28(9.4 \%)$ & $33(11.3 \%)$ \\
\hline CABG & $3(1.0 \%)$ & $5(1.7 \%)$ & $5(1.7 \%)$ & $5(1.7 \%)$ & $5(1.7 \%)$ \\
\hline Ischaemia-driven TVR & $24(8.0 \%)$ & $31(10.3 \%)$ & $36(12.1 \%)$ & $39(13.1 \%)$ & 45 (15.5\%) \\
\hline $\mathrm{PCl}$ & $21(7.0 \%)$ & $28(9.3 \%)$ & $33(11.1 \%)$ & $36(12.1 \%)$ & $41(14.1 \%)$ \\
\hline CABG & $3(1.0 \%)$ & $5(1.7 \%)$ & $5(1.7 \%)$ & $5(1.7 \%)$ & $6(2.1 \%)$ \\
\hline Target vessel failure & $34(11.3 \%)$ & $45(15.0 \%)$ & $51(17.1 \%)$ & $57(19.2 \%)$ & $62(21.3 \%)$ \\
\hline \multicolumn{6}{|l|}{ Stent thrombosis (ARC def.) } \\
\hline Definite or probable & $3(1.0 \%)$ & $6(2.0 \%)$ & $7(2.3 \%)$ & $7(2.4 \%)$ & $9(3.1 \%)$ \\
\hline Possible & $0(0.0 \%)$ & $1(0.3 \%)$ & $3(1.0 \%)$ & $6(2.0 \%)$ & $6(2.1 \%)$ \\
\hline
\end{tabular}

